\title{
EXPERIÊNCIA INICIAL NO TRATAMENTO ENDOSCÓPICO DE FÍSTULAS GASTROCUTÂNEAS PÓS-GASTROPLASTIA VERTICAL REDUTORA ATRAVÉS DA APLICAÇÃO DE MATRIZ ACELULAR FIBROGÊNICA
}

\author{
Fauze MALUF-FILHO ${ }^{1,2}$, Marcelo Simas de LIMAA, ${ }^{1,}$, Fabio HONDO', \\ José Humberto GIORDANO-NAPPI ${ }^{1}$, Teresa GARRIDO ${ }^{1}$ e Paulo SAKAI ${ }^{1}$
}

\begin{abstract}
RESUMO - Racional - A gastroplastia redutora à Capella, que combina técnicas restritiva e disabsortiva, é o procedimento bariátrico mais realizado no Brasil. Uma das complicações mais temidas desta operação é a fístula gastrocutânea que ocorre na linha de grampeamento vertical da neocâmara. $\mathrm{O}$ tratamento inicial consiste em drenagem adequada, suporte nutricional, bloqueio da produção de ácido e antibioticoterapia. Em $20 \%$ a $30 \%$ dos casos, a fístula tomará curso crônico. Nestes casos, vários tratamentos endoscópicos têm sido sugeridos. Objetivo - Descrever a técnica e os resultados do tratamento endoscópico das fístulas gastrocutâneas decorrentes de operações bariátricas através da aplicação de matriz acelular fibrogênica. Métodos - Nesta série de casos, descrevem-se 25 pacientes com esta complicação tratados de maneira pioneira através da aplicação endoscópica de matriz acelular fibrogênica. O tempo entre o diagnóstico da fístula e a primeira sessão do tratamento endoscópico variou de 4 a 25 semanas, mediana: 7 semanas). Resultados - Dos 25 pacientes, 20 (80\%) tiveram a fístula fechada pelo método. Seis deles com uma única sessão $(30,0 \%), 11$ necessitaram de duas aplicações $(55,0 \%)$ e 3 pacientes, de uma terceira sessão $(15,0 \%)$ para a obliteração do trajeto fistuloso. Não houve complicações relacionadas ao procedimento. Conclusão - O uso de matriz fibrogênica para o tratamento endoscópico da fístula gástrica após operação de Capella é método seguro e eficaz, sendo sua principal limitação o número de sessões necessárias.
\end{abstract}

DESCRITORES - Fístula cutânea. Fístula gástrica. Gastroplastia. Obesidade mórbida. Endoscopia. Matriz extracelular. Materiais biocompatíveis.

\section{INTRODUÇÃO}

A gastroplastia redutora à Capella, que combina técnicas restritiva e disabsortiva, é o procedimento bariátrico mais realizado no Brasil e Estados Unidos ${ }^{(4,11)}$. A padronização dos tempos cirúrgicos, a perda ponderal média de $40 \%$ do peso inicial e a menor incidência de distúrbios eletrolíticos garantiram sua difusão entre pacientes e cirurgiões ${ }^{(9,10,16)}$.

A despeito de toda a evolução da técnica e do material cirúrgico, mormente o emprego dos grampeadores lineares cortantes, ainda há morbidade e mortalidade relacionadas a esta operação bariátrica ${ }^{(3)}$.

A fístula gastrocutânea, junto ao grampeamento do ângulo esôfago-gástrico, está entre as complicações mais freqüentes, diagnosticada em $0,5 \%$ até $8,4 \%$ dos operados, em diferentes séries. Também figura entre as complicações de difícil tratamento, por vezes exigindo reintervenções operatórias, com taxas de sucesso variáveis, acrescentando morbidade a enfermo freqüentemente com afecções cardiológicas, respiratórias e metabólicas ${ }^{(3)}$.

$\mathrm{O}$ tratamento desta complicação envolve a reoperação com lavagem e desbridamento da região da fístula, exposição do local e ressutura, quando a reintervenção é precoce e a lesão favorável. A retirada do elemento de restrição, no caso o anel, é mandatória quando a deiscência ocorre acima dele, podendo haver dano aos resultados de perda ponderal em longo prazo. A redrenagem e gastrostomia no estômago excluso completam a reoperação.

Diante deste quadro, são desejáveis técnicas alternativas, menos invasivas, de tratamento da fístula gastrocutânea, pós-gastroplastia vertical redutora tipo Capella. De fato, têm-se descritos diferentes métodos endoscópicos, visando a correção das fístulas pós-operatórias. Na literatura surgem relatos de casos em que foram empregados clipes endoscópicos, cola de fibrina e, mais recentemente, o SurgiSIS $^{\circledR}(\text { Cook, WS, NC) })^{(5,6,8,13,17,18,19,20,21)}$. Este material

'Serviço de Endoscopia Gastrointestinal do Hospital das Clínicas da Faculdade de Medicina da Universidade de São Paulo; ${ }^{2}$ Serviço de Endoscopia da Real e Benemérita Sociedade Portuguesa de Beneficência de São Paulo - Hospital São Joaquim, São Paulo, SP.

Correspondência: Dr. Fauze Maluf-Filho - Rua Olegário Mariano, 488 - 05612-000 - São Paulo, SP. fauze.maluf@terra.com.br 
consiste em matriz acelular, produzida a partir da submucosa do intestino delgado porcino. Estimula a taxia celular, dando suporte para a proliferação e formação de verdadeira rede de fibroblastos, sendo, posteriormente, incorporada pela cicatriz, sem estimular reação inflamatória de corpo estranho ${ }^{(12)}$.

Objetivou-se apresentar experiência inicial de 25 casos em que se aplicou endoscopicamente este material com o intuito de corrigir tais fístulas gastrocutâneas, prescindindo de reintervenção operatória.

\section{MÉTODO}

Todos os pacientes que evoluíram com débito salivar pelo dreno abdominal ou com necessidade de reoperação precoce por deiscência da linha de grampeamento foram submetidos a teste de ingestão de azul de metileno, exame radiológico com contraste iodado e endoscopia digestiva diagnóstica para confirmação e documentação do orifício fistuloso.

Iniciado o tratamento conservador através da administração de antibioticoterapia, inibidor de bomba de prótons, nutrição parenteral ou enteral (através de gastrostomia ou sonda de Dubbhoff) ou, em alguns casos, com o reposicionamento do dreno e retirada do anel restritivo. Após 4 semanas, foram repetidos os exames, incluindo a reavaliação endoscópica. Nos casos em que não houve qualquer alteração do aspecto endoscópico da fístula e do seu débito, optouse pela inclusão do paciente no protocolo (Figura 1).

De abril de 2001 a março de 2007, foram incluídos 25 pacientes (idades: 25 a 61 anos; sexo: 14 fem, 11 masc), com orifício fistuloso variando de 5 a $20 \mathrm{~mm}$ de diâmetro. O tempo entre o diagnóstico da fístula e a primeira sessão do tratamento endoscópico variou de 4 a 25 semanas, mediana: 7 semanas). Os pacientes foram encaminhados para o Serviço de Endoscopia do Hospital São Joaquim da Beneficência Portuguesa de São Paulo (BPSP) e submetidos ao tratamento apenas após leitura e assinatura do consentimento informado. Este estudo foi aprovado pela Comissão de Ética da BPSP.

O SurgiSIS ${ }^{\circledR}$ é comercializado em diferentes formas e tamanhos. Para os primeiros 20 enfermos, foram aplicadas tiras com $7 \mathrm{~cm}$ de comprimento por $0,6 \mathrm{~cm}$ de largura. Após embebição em solução salina estéril, durante 5 minutos, realizou-se divisão da tira em duas peças de $3,5 \mathrm{~cm}$ de comprimento para que fosse possível a adaptação de cada metade na alça de polipectomia. Foi realizado exame endoscópico sob sedação consciente e anestesia tópica de orofaringe. Identificado o orifício fistuloso, a alça de polipectomia foi inserida no interior do mesmo. Empurrando-se a alça, conseguiu-se colocar o SurgiSIS ${ }^{\circledR}$ no interior do trajeto fistuloso.

Para os últimos cinco pacientes, utilizou-se a recémlançada apresentação em forma de cone (Figura 2), com $9 \mathrm{~cm}$ de comprimento e $10 \mathrm{~mm}$ de base na extremidade mais larga (SurgiSIS ${ }^{\circledR}$-AFP). Para a aplicação desta última, é necessária a cateterização retrógrada do trajeto fistuloso com cateter teflonado de 7Fr. Acopla-se a extremidade afilada do SurgiSIS ${ }^{\circledR}$ no lúmen do cateter (Figura 3), que é tracionado pelo orifício cutâneo da fístula, até que o cone ocupe todo o trajeto fistuloso (Figura 4).

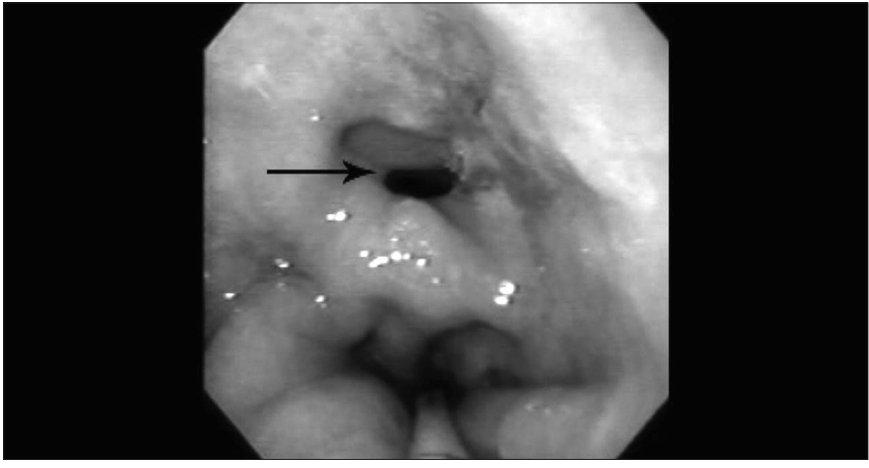

FIGURA 1. Aspecto endoscópico de fístula gastrocutânea

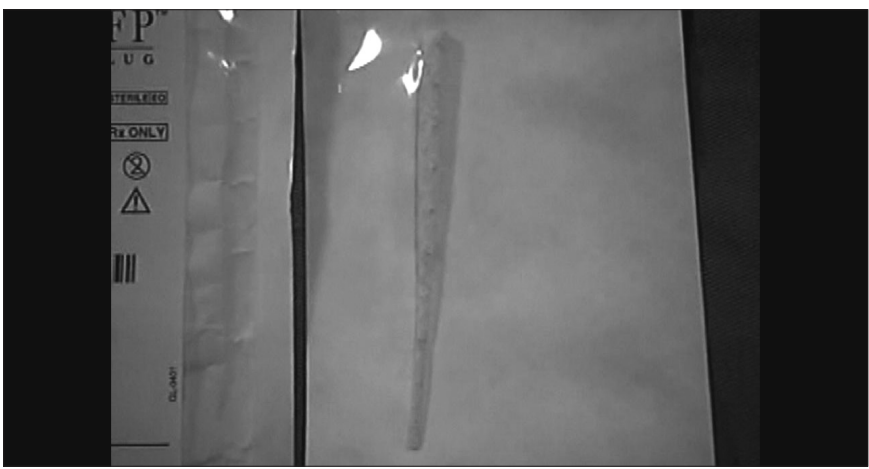

FIGURA 2. A matriz acelular na apresentação em forma de cone

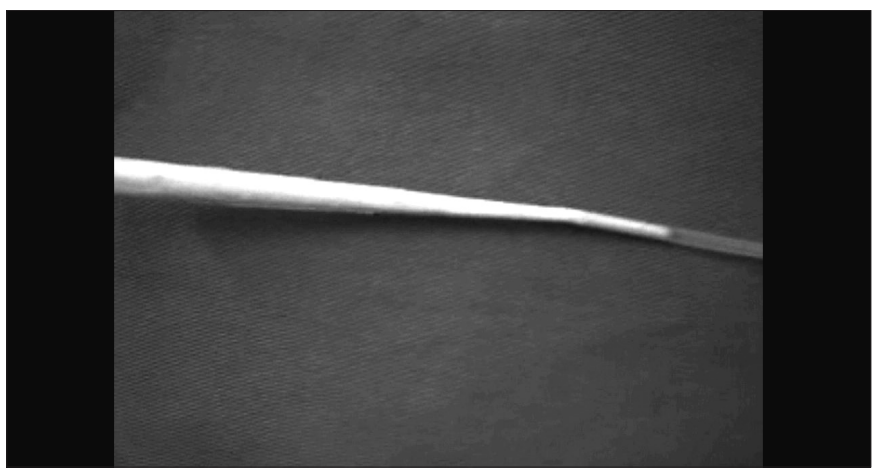

FIGURA 3. Aspecto da montagem do material em cateter teflonado

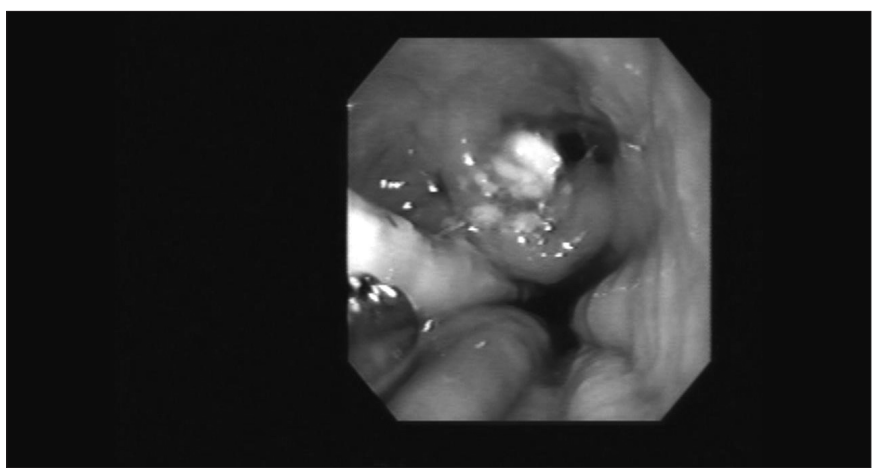

FIGURA 4. Aspecto endoscópico do material ocluindo o orifício fistuloso 
As aplicações foram feitas com intervalos de 3 semanas, sendo sempre precedidas de nova rotina de exames. Três semanas após a terceira sessão a persistência da fístula foi considerada como falha terapêutica.

\section{RESULTADOS}

Dos 25 pacientes, $20(80 \%)$ tiveram a fístula fechada pelo método. Seis deles com uma única sessão $(30,0 \%), 11$ necessitaram de duas aplicações $(55,0 \%)$ e 3 pacientes, de uma terceira sessão $(15,0 \%)$ para a obliteração do trajeto fistuloso (Figura 5). Em cinco casos, houve falha terapêutica documentada após a terceira semana da terceira sessão, porém em todos eles notou-se clara redução do orifício fistuloso, documentada fotograficamente. Estes pacientes foram submetidos ao tratamento cirúrgico da fístula. Em nenhum deles foram encontradas lojas ou coleções com a matriz acelular em seu interior.

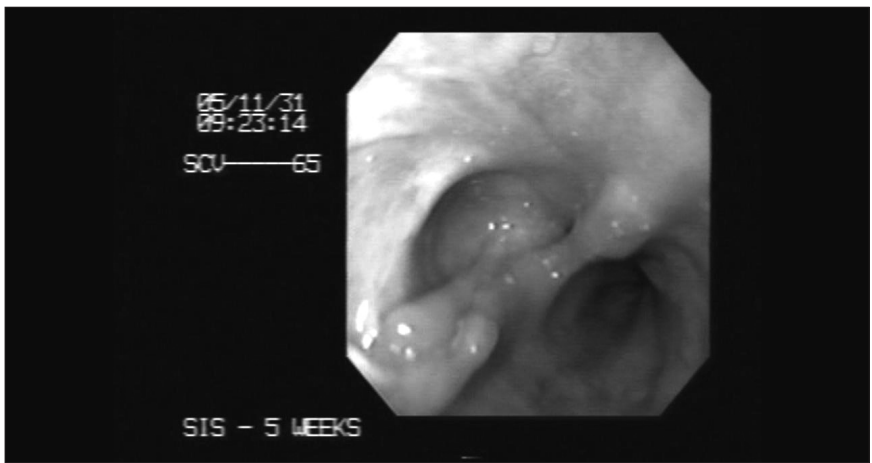

FIGURA 5. Aspecto endoscópico final com fechamento do orifício fistuloso

\section{DISCUSSÃO}

É consenso na Sociedade Brasileira de Cirurgia Bariátrica, a segunda maior em todo o mundo em número de associados dentre as agremiações que congregam cirugiões bariátricos, que qualquer centro que realize tais procedimentos deve manter índices de mortalidade inferiores a $2 \%$.

É claro que, para tanto, se faz fundamental o controle das co-morbidades no período pré-operatório. Mas tratar-se de procedimento eletivo não afasta de vez as complicações e, quando elas ocorrem, saber conduzir a terapêutica e controlar a ansiedade gerada no paciente são os diferenciais de qualidade que se querem oferecer.

A conhecida evolução clínica das complicações no paciente obeso, que inicialmente é silenciosa, exige elevado grau de suspeição diagnóstica e prontidão na realização de exames diagnósticos e no tratamento.

Se o papel diagnóstico da endoscopia digestiva já está estabelecido, ainda se faz necessário o desenvolvimento e a propagação de técnicas terapêuticas para o tratamento das complicações da cirurgia bariátrica.
O obeso tem suas particularidades e também particular é o procedimento de Capella. Muito se discute sobre a causa das fístulas relacionadas a esta técnica. Sabe-se que a linha de grampeamento, particularmente em sua extremidade cranial, é o local mais suscetível a tal complicação. Este fato deve ser explicado pelo ciclo isquemia, seguida de necrose, digestão péptica e infecção. Historicamente, a técnica, assim como o material cirúrgico, evoluíram. O sepultamento da linha de grampeamento, a interposição de alça jejunal entre as câmaras gástricas, a realização de gastrostomia e o teste intraoperatório da anastomose são exemplos dessa evolução. Ainda assim, estima-se que a fístula gastrocutânea, resultante da gastroplastia vertical redutora à Fobi-Capella ocorra, em média, em 2\% dos pacientes operados. Instituído o tratamento clínico, comum às fístulas pós-operatórias, espera-se a resolução desta complicação em até $80 \%$ dos casos. Para os demais casos, a endoscopia terapêutica tem oferecido diferentes métodos para controle desta morbidade pós-operatória. A este respeito, as séries são bastante limitadas. Há a descrição de três pacientes tratados com sucesso, através da aplicação endoscópica de selante de fibrina no orifício de fístulas gastrocutâneas decorrentes de operações bariátricas. Destaca-se que em um deles, houve necessidade de nove sessões do tratamento endoscópico ${ }^{(18)}$. Em outra série de três casos, os autores manejaram endoscopicamente com sucesso as fístulas gastrocutâneas, associando a termocoagulação com plasma de argônio, seguida da aplicação de clips metálicos, selante de fibrina e prótese plástica autoexpansível ${ }^{(15)}$. A análise destes estudos revela o caráter persistente deste tipo particular de fístula o que exige, não raro, várias sessões do tratamento endoscópico proposto.

Embora existam descrições da aplicação do SurgiSIS ${ }^{\circledR}$ para o reparo de hérnias da parede abdominal ${ }^{(7)}$, para a substituição da dura-máter, como curativo de úlceras varicosas em membros inferiores, para correção de extrofia vesical e cistoplastia ${ }^{(1)}$ é pioneira a introdução desta técnica para o tratamento de fístulas resultantes da gastroplastia vertical redutora ${ }^{(13,14)}$. A versatilidade da aplicação clínica deste material reflete sua capacidade de estimular a cicatrização, com mínima reação inflamatória, sem rejeição. De fato, no modelo experimental em ratos Wistar, a comparação entre a reação suscitada pelo implante abdominal de telas feitas de polipropileno ou de SurgiSIS ${ }^{\circledR}$ revela igual deposição de colágeno, força tênsil semelhante, com menor reação inflamatória e aderências quando do emprego do material biológico ${ }^{(12)}$. Recentemente, foi estudada a reação imunológica ao SurgiSIS ${ }^{\circledR}$ em pacientes submetidos a implante para correção de hérnia inguinal. Em todos, foram detectados anticorpos específicos cujos títulos foram diminuindo a partir dos 6 meses da operação. Em nenhum deles, houve sinais imunológicos ou clínicos de rejeição do implante ${ }^{(2)}$.

$\mathrm{Na}$ fase inicial deste estudo, em que se utilizou a matriz em forma de tiras, o sucesso foi menor, de $75 \%$, tendo sido necessárias duas ou três sessões na maioria dos casos. Por outro lado, nos últimos cinco casos em que se aplicou a forma cônica da matriz, obteve-se o fechamento da fístula em todo eles, em uma única sessão. Ressalta-se que há experiências anedóticas em que outras fístulas pós-operatórias foram curadas através de várias (mais de cinco) sessões de aplicação da matriz acelular.

Vale a pena destacar que todos os cinco casos de insucessos foram resolvidos através do tratamento operatório. Nestes casos, as informações obtidas com os cirurgiões foram de que o trajeto 
fistuloso era tortuoso, com várias saculações infectadas, levantando possível explicação para a falha do tratamento endoscópico.

\section{CONCLUSÃO}

Os métodos endoscópicos continuam evoluindo, juntamente com a cirurgia e com os novos desafios que se impõem.
A cirurgia bariátrica pode ser beneficiada pela endoscopia no controle da morbidade inerente à técnica.

O método, aqui apresentado em casuística inicial, demonstrou ser seguro e efetivo.

É clara a noção de que a aplicação endoscópica da matriz acelular é mais um dos métodos adjuvantes no tratamento da fístula resultante da gastroplastia vertical redutora.

Maluf-Filho F, Lima MS, Hondo F, Giordano-Nappi JH, Garrido T, Sakai P. Endoscopic placement of a "plug" made of acellular biomaterial: a new technique for the repair of gastric leak after Roux-en-Y gastric bypass. Arq Gastroenterol. 2008;45(3):208-11.

ABSTRACT - Background - Roux-en-Y gastric bypass is one of the most commonly performed bariatric surgeries in Brazil. Gastric leaks are relatively uncommon and potentially dangerous complications. The initial management of gastric leaks consists in adequate drainage, nutritional support, antibiotics and acid suppression. In almost 30\% of the cases the fistula will become chronic. Aim - A novel peroral endoscopic treatment of gastric leaks in Roux-en-Y gastric bypassed patients is presented. Methods - An acellular biomaterial was endocopically placed in the fistulous orifice in order to promote healing and avoid surgery in 25 patients. The time between fistula diagnosis and endoscopic treatment varied from 4 to 25 weeks (median: 7 weeks). Results - Endoscopic treatment was successful in $20(80 \%)$ patients. Fistula closure was obtained after one, two and three sessions in $6(30 \%), 11(55 \%)$ and $3(15 \%)$ patients, respectively. No procedure related complications were observed. Conclusion - Endoscopic repair of gastric leak after Roux-en-Y gastric bypass by using an acellular biomaterial is safe and effective. However two or three endoscopic sessions are usually needed.

HEADINGS - Cutaneous fistula. Gastric fistula. Gastroplasty. Obesity, morbid. Endoscopy. Extracellular matrix. Biocompatible materials.

\section{REFERÊNCIAS}

1. Alpert SA, Cheng EY, Kaplan WE, Snodglrass WT, Wilcox DT, Kropp BP. Bladder neck fistula after the complete primary repair of exstrophy: a multi-institutional experience. J Urol. 2005; 174:1687-89.

2. Ansaloni L, Cambrini P, Catena F, Di Saverio S, Gagliardi S, Gazzotti F, Hodde JP, Metzger DW, D'Alessandro L, Pinna AD. Immune response to small intestinal submucosa (surgisis) implant in humans: preliminary observations. J Invest Surg. 2007;20:237-41.

3. Barroso FL, Alonso ADS, Leite MA. Complicações cirúrgicas intra- operatórias e do pós- operatório recente. In: Barroso FL. Cirurgia da obesidade. São Paulo: Atheneu; 2002.

4. Capella RF, Capella JF, Mandaec H, Nath P. Vertical banded gastroplasty - gastric bypass: preliminary report. Obes Surg. 1991;1:389-95.

5. Cellier C, Landi B, Faye A, Wind P, Frileux P, Cugnenc PH, Barbier JP. Uppe gastrointestinal tract fistulae: endoscopic obliteration with fibrin sealant. Gastrointest Endosc. 1996;44:731-3.

6. Dunn CJ, Goa KL. Fibrin sealant: a review of its use in surgery and endoscopy. Drugs. 1999;58:863-86.

7. Edelman DS, Hodde JP. Bioactive prosthetic material for treatment of hernias. Surg Technol Int. 2006;15:104-8.

8. Familiari P, Macri A, Consolo P, Angio L, Scaffidi MG, Famulari C, Familiari L. Endoscopic clipping of a colocutaneous fistula following necrotizing pancreatitis: case report. Dig Liver Dis. 2003;35:907-10.

9. Fobi MA, Lee H, Flemming A. The surgical technique of the banded Roux in Y gastric bypass. J Obesity Weight Reg. 1989;8:99-102,

10. Fobi MA, Lee H, Holness R, Cabinda D. Gastric bypass operation for obesity. World J Surg. 1998;22:925-35

11. Garrido Jr AB. Cirurgia da obesidade. São Paulo: Atheneu; 2001. p.327.

12. Konstantinovic ML, Lagae P, Zheng F, Verbeken EK, De Ridder D, Deprest JA Comparison of host response to polyprolpylene and non-cross-linked porcine small intestine serosal-derived collagen implants in rat model. BJOG. 2005;112:1554-60.

13. Maluf-Filho F, Garrido T, Pitelli J, Moura EGH, Berti LV, Garrido Jr AB, Sakai P, Ishioka S, Gama Rodrigues JJ. Endoscopic treatment of esophagogastric fistulae with an acellular matrix: a pilot study. Gastrointest Endosc. 2002;55:pAB 108-8.

14. Maluf-Filho F, Moura E, Sakai P, Garrido AB, Ishioka S, Gama Rodrigues J, Berti L. Endoscopic treatment of esophagogastric fistulae with an acellular matrix. Gastrointest Endosc. 2004;59:pAB151-1

15. Merrifield BF, Lautz D, Thompson CC Endoscopic repairs of gastric leak after Roux-en-Y gastric bypass: a less invasive approach. Gastrointest Endosc. 2006;63:710-4.

16. Murr MM, Balsinger BM, Kennedy F, Mai JL, Sarr MG:. Malabsorptive procedures for severe obesity: comparison of pancreaticobiliary bypass and very very long limb Roux-en-Y gastric bypass. J Gastrointest Surg. 1999;3:607-12,

17. Papavramidis ST, Eleftheriadis EE, Apostolidis DN, Kotzampassi KE. Endoscopic fibrin sealing of high-output non-healing gastrocutaneous fistulas after vertical gastroplasty in morbidly obese patients. Obes Surg. 2001;11:766-9.

18. Papavramidis ST, Eleftheriadis EE, Papavramidis TS, Kotzampassi KE, Gamvros OG Endoscopic management of gastrocutaneos fistula after bariatric surgery by using a fibrin sealant. Gastrointest Endosc. 2004:59:296-300.

19. Rabago LR, Ventosa N, Castro JL, Marco J, Herrera N, Gea F. Endoscopic treatment of postoperative fistulas resistant to conservative management using biological fibrin glue. Endoscopy. 2002;34:632-8

20. Shand A, Pendlebury J, Reading S, Papachrysostomou M, Ghosh S. Endoscopic fibrin sealant injection: a novel method of closing a refractory gastrocutaneous fistula. Gastrointest Endosc. 1997;46:357-8.

21. Thurairajah P, Hawthorne AB. Endoscopic clipping of a nonhealing gastrocutaneous fistula following gastrostomy removal. Endoscopy. 2004;36:834. 